\title{
Morphometric Analysis of Hippocampal Pyramidal Neurons in situ and in Grafts Developing in the Anterior Eye Chambers of Young and Aged Wistar Rats
}

\author{
Z.N. Zhuravleva, V.N. Saifullina and C.I. Zenchenko \\ Institute of Theoretical and Experimental Biophysics, Russian Academy of Sciences, \\ Puschino, Moscow Distr., Russia
}

\section{SUMMARY}

We performed a morphometric analysis of the somatic and nuclear areas in the pyramidal neurons of the hippocampal fields CA1 and CA3 in situ and in grafts developing for six weeks in the anterior eye chambers of young (3-to-9 wk.) and of aged (18-to-19.5 mos.) Wistar rats. The mean areas of the CA1 pyramidal somata and nuclei were significantly decreased in the aged animals in situ. The mean parameters of the CA3 pyramidal neurons were not changed, although their distribution was different (bimodal versus unimodal in the young animals). In both groups of recipients, the areas of CA1 neurons and of their nuclei were significantly larger in the grafted tissue than those found in situ. The areas of CA3 neurons did not show any difference in aged recipients and demonstrated only slight hypertrophy in young recipients. We concluded that the area sizes of the pyramidal cell bodies and nuclei in CA1 neurons are more sensitive than those of CA3 neurons to both aging and transplantation. The age of recipients did not significantly influence the growth and development of grafted pyramidal cells.

\section{KEY WORDS}

hippocampus, intraocular grafts, aging, neural transplantation

\footnotetext{
Reprint address:

Z.N. Zhuravleva

Institute of Theoretical and Experimental Biophysics

Russian Academy of Sciences

Puschino, Moscow Distr., Russia, 142292
}

\section{INTRODUCTION}

Transplanted embryonic nervous tissue in the anterior eye chamber provides a convenient model for investigating the development of neurons in conditions of isolation from other brain structures. In the anterior eye chamber, the grafted tissue receives innervation as well as the supply from peripheral nerves and blood vessels ingrowing from the iris of the recipient. The graft is surrounded by intraocular fluid, the composition of which is similar to that of cerebro-spinal fluid. Intraocular grafts are useful for investigating the comparative role of various factors in the growth and development of neurons: their genetic potential, nervous surrounding, and the general internal environment provided by the recipient.

In previous research, we investigated the growth and shape of intraocular hippocampal grafts in young and aged recipient rats $/ 28 /$. We now present data on the morphometric analysis of pyramidal neurons in two main hippocampal areas (CA1 and CA3) in both age groups. The results were compared with similar data on the hippocampus of the recipient animals. Despite multiple technical difficulties and skepticism, transplantation of nervous tissue is considered a possible therapeutic approach to correcting brain pathology in humans, while stressing the necessity for further basic research on developing safe and successful methods $19,10,11,17,26 /$. As neurological and neurodegenerative disorders are more frequently encountered in the aged brain than in the young brain, one important question to be answered is whether embryonic nerve cells would become involved in an accelerated process of aging under the influence of the changed internal medium of the aging organism. 


\section{METHODS}

Animals

Rats of the Wistar strain were used in these experiments. Donor-rat embryos were taken on days 17 and 18 of gestation (E17-18). Recipient male rats were divided into two age groups, each containing three animals: (1) young (21-d-old, $\sim 150 \mathrm{~g}$ ) and (2) aged (18-mo-old, 300-400 g).

\section{Tissue preparation}

Caesarean section was performed in sterile conditions under deep penthobarbital anesthesia (40 mg/kg). The embryo was first washed in Eagle's solution, and then the brain was exposed. The hippocampal tissue containing fields CA1-CA3 (volume about $2 \mathrm{~mm}$ ), without dentate fascia, was dissected under a stereomicroscope and placed into cooled Eagle's solution. A drop of atropine was placed upon the eye of Penthobarbital-anesthetized recipients, and local anesthesia of the eye (dicaine, $2 \%$ ) was applied. The cornea was incised, and embryonic hippocampal tissue was injected into the anterior eye chamber with the help of a syringe with a glass capillary. Six weeks later, the animals were sacrificed by administering an overdose of Penthobarbital. The grafts were taken out together with the region of iris upon which they developed, and simultaneously the pieces of the recipients' hippocampal areas CA1 and CA3 were dissected. The grafts and the recipients' hippocampi were fixed in Carnoy medium followed by embedding into paraffin. Histological sections were stained by mixture of gallocyanine and chromium alum according to the Einarson method.

\section{Morphometric analysis}

Morphometric analysis was performed on the pyramidal neurons of the CA1 and CA3 areas in situ and in grafts of young (9-wk-old) and aged (19.5-mo-old) recipients. Photomicrographs of the cells and of object-micrometer were made under standard magnification (object glass 40x, ocular $7 x$ ). For each animal, 100 well-defined pyramidal neurons with clear central nuclear profiles were selected in CA1 and CA3 of the grafts and in the hippocampus in situ. The negative images were analyzed using the computerized system Image Analysis. The program (author V.I. Hachko) allowed us to obtain cross-section areas (in $\mathrm{mkm}$ ) of neuronal perikarya and nuclei and their means for each block of experimental data. The distribution of individual neuronal sizes for each group is presented in histograms. On the whole, 300 pyramidal cells were measured in each of eight experimental blocks. Comparisons were made between (a) the CA1 and CA3 neurons; (b) grafted and in situ neurons; and (c) young and aged animals. The Student's t-test was used for statistical evaluation.

\section{RESULTS}

Comparison of CA1 and CA3 pyramidal cells in the hippocampus of young and aged animals

The data in Table 1 demonstrate the well-known differences between the dimensions of $\mathrm{CAl}$ and CA3 pyramidal neurons. The significantly greater sizes of the bodies and the wider distribution of pyramidal cells indicated a greater variability of such cells in CA3 area, and comparing their nuclear dimensions confirmed this conclusion.

The mean areas of the CAl pyramidal neurons in aged rats were significantly smaller than those in young rats. In aged animals, the mean area of cell profiles was smaller by $14.4 \%$, and the nuclear profiles were smaller by $9.1 \%$. Analysis of the histograms showed an increased number of small neurons. Whereas in young rats the maximum in the histogram was in the range of 150 to $200 \mathrm{mkm}^{2}$, the

Table 1

Hippocampal CA1 and CA3 pyramidal neurons in situ and in intraocular grafts in young and aged rats: Mean area of cell bodies and nuclei $\left(\mathrm{mkm}^{2},(\mathrm{x}+\mathrm{sx})\right.$

\begin{tabular}{ccccc}
\hline Location & \multicolumn{2}{c}{ Cell Body } & \multicolumn{3}{c}{ Nucleus } \\
\hline In situ & Young & Aged & Young & Aged \\
\cline { 2 - 5 } CA1 & $189.4 \pm 1.8$ & $165.5 \pm 1.6^{\mathrm{a}}$ & $91.9 \pm 0.7$ & $84.2 \pm 1.4^{\mathrm{a}}$ \\
CA3 & $376.2 \pm 4.0$ & $380.2 \pm 5.0$ & $138.2 \pm 1.2$ & $141.3 \pm 1.6$ \\
In oculo & & & & \\
CA1 & $212.3 \pm 1.5^{\mathrm{a}}$ & $211.4 \pm 2.0^{\mathrm{b}}$ & $103.0 \pm 0.7^{\mathrm{b}}$ & $97.1 \pm 1.5^{\mathrm{a}, \mathrm{b}}$ \\
CA3 & $398.8 \pm 5.2^{\mathrm{b}}$ & $379.0 \pm 4.2$ & $140.1 \pm 1.2$ & $137.3 \pm 1.2$ \\
\hline
\end{tabular}

${ }^{\mathrm{a}}$ Significant difference, aged versus young animals;

${ }^{b}$ Significant difference, in oculo graft versus in situ hippocampus 
maximum in aged rats was shifted to the range of 125-175 $\mathrm{mkm}^{2}$. The compact, unimodal type of distribution, however, was preserved.

For both age groups, the mean sizes of the somatic and nuclear profiles of the $\mathrm{CA} 3$ areas did not show statistically significant differences. But nevertheless, certain changes in the type of distribution of neuronal sizes were obvious. In aged animals, the distribution was bimodal. A rather wide maximum-typical for $\mathrm{CA} 3$ neurons in young rats, with the center at $375 \mathrm{mkm}^{2}$ - was substituted by two maxima in the ranges of 300 and $425 \mathrm{mkm}^{2}$.

\section{Cytological characteristics of hippocampal grafts}

In both age groups, the hippocampal grafts survived and developed equally well. Pathologic infiltration of the tissue by mononuclear cells did not occur, although we noticed that in two aged recipients, the number of lymphocytes increased inside the large vessels that were growing into graft from the host iris.

Additionally, we saw no qualitative difference in cytoarchitectonics of the grafts in the two age groups. Usually the grafts had an elongated shape, with the longitudinal axis up to $2-4 \mathrm{~mm}$. The cellular layer, resembling that of the hippocampus in situ, contained well-identifiable CA1 and CA3 pyramidal neurons that had typical differences in the organization of apical dendrites, which in CA3 neurons had bifurcation close to the neuronal bodies.

Photomicrographs of pyramidal cells (Fig. 1) showed that the organization of $\mathrm{CA} 3$ neurons in the layer was less compact than that of $\mathrm{CAl}$ pyramidal cells. As a rule, pyramidal neurons with the typical characteristics of CA1 and CA3 were spatially segregated. The apical dendrites were oriented predominantly toward the iris, although the grafts had not been specifically oriented when injected into anterior eye chamber. The dimensions and the degree of organization of the cellular layer varied individually. Pyramidal neurons that were displaced from the layer were not used for morphometric analysis. The structure of the nuclei and cytoplasm of the grafted neurons was well developed, with clearly delineated cellular membranes, basophylic nucleoli, chromatin at the nuclear periphery, and tigroid clamps in the cytoplasm. In one graft from a young recipient, some neurons with vacuolized cytoplasm were observed; whereas dark pyramidal neurons were encountered in another graft from an aged recipient. It should be noted, that the both grafts were among the most developed, large ones.

\section{In situ hippocampal neurons versus grafted neurons}

In grafts of both recipient age groups, the mean areas of CAl pyramidal profiles were significantly enlarged. The mean area of the cell bodies in the grafts of young recipients was $11.6 \%$ larger and that of the nuclei was $12.1 \%$ larger than the corresponding parameters of their own hippocampal pyramidal neurons in situ. The difference was even more prominent in the aged recipients, where the corresponding difference was $27.7 \%$ for cell bodies and $15.3 \%$ for nuclei. Clearly, this difference resulted from the significant reduction in the size of CA1 neurons of the aged recipients that were used for comparison.

The histograms in Fig. 2 show the changed distribution of the grafted CAl cells. Although all histograms had a similar unimodal shape, the maxima for the grafted cells in both young and aged recipients were shifted to the right. In the young recipients, however, the population of grafted pyramidal neurons was more uniform than that in the aged recipients, and many neurons were grouped at the narrow maximum around $225 \mathrm{mkm}$.

The situation was different for grafted CA3 pyramidal neurons. The only statistically significant difference was observed in cell bodies developing in the anterior eye chamber of the young recipients, but even this difference was much smaller than for the CA1 neurons developing in the same conditions (CA3 increased by $6.5 \%$ versus $11.6 \%$ for $\mathrm{CA} 1$ ).

The distribution of grafted $\mathrm{CA} 3$ neurons in young recipients had the same maximum as the histogram of cell sizes in situ $(375 \mathrm{mkm})$; only a limited group of grafted neurons had sizes in the range of 575-775 mkm; such neurons were absent in situ. The areas of the CA3 neuronal nuclei in grafts in young recipients-as well as the sizes of both cell bodies and nuclei in aged recipients-did not differ from the corresponding parameters of in situ neurons. 


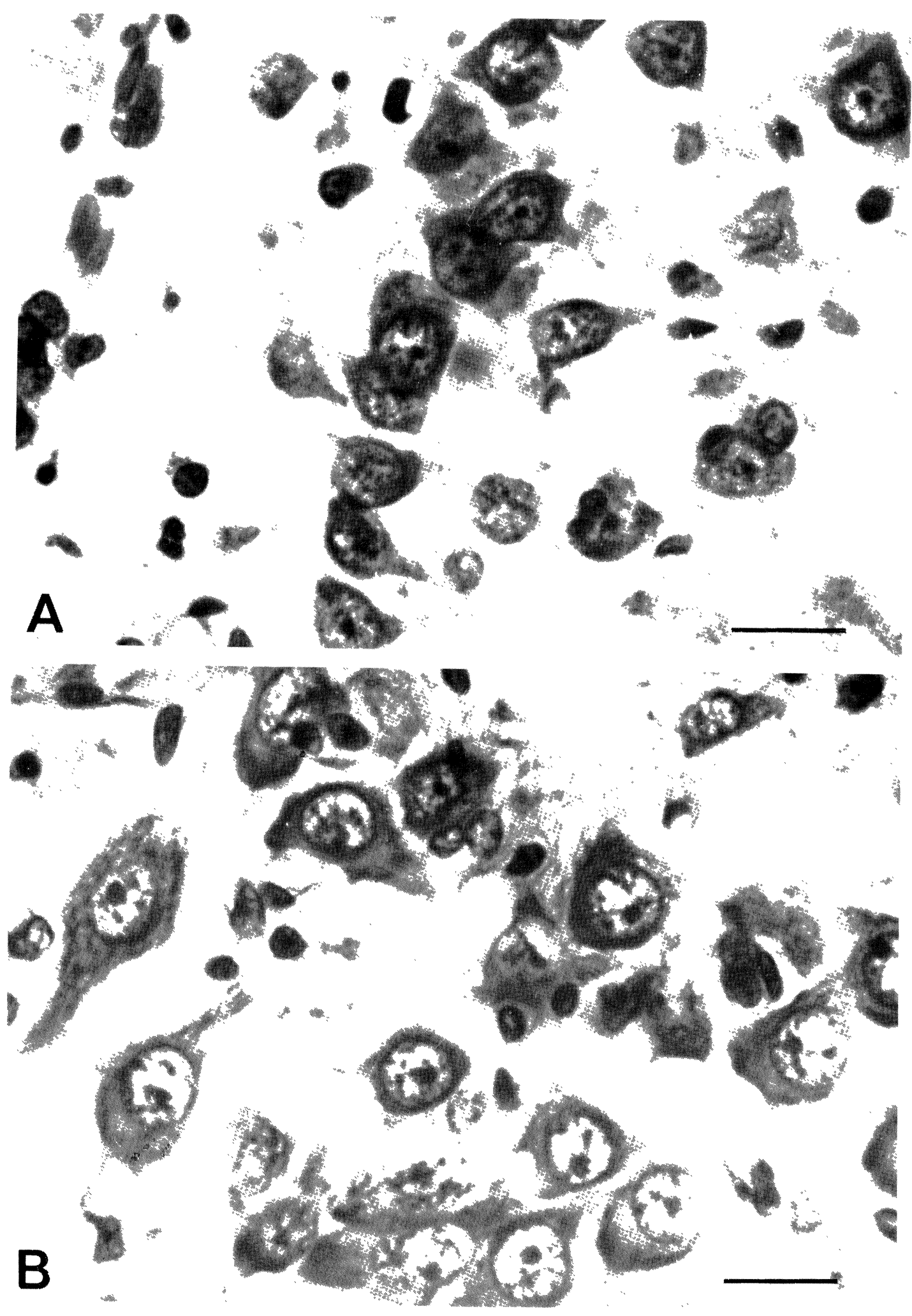

Fig. 1. Photomicrographs of CA1 (A) and CA3 (B) pyramidal neurons of intraocular hippocampal grafts. Scale $-25 \mathrm{mkm}$. 

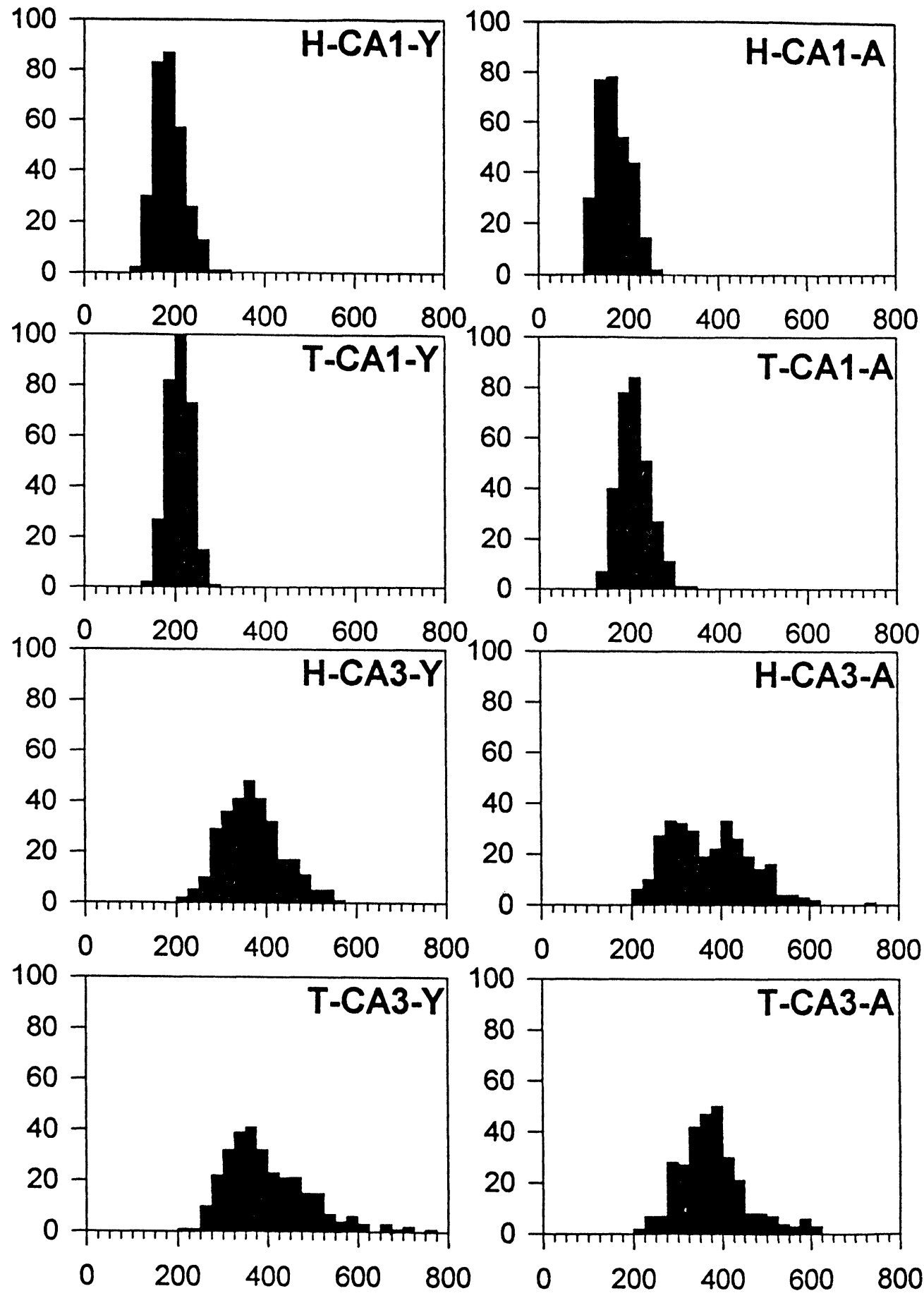

Fig. 2. Distribution of CA1 and CA3 pyramidal cells in the in situ hippocampus and in the in oculo grafts in young and aged rats. Abscissa-cell body sizes in $\mathrm{mkm}$; ordinate-number of the measured cells. Indexes: H-hippocampus in situ; T-hippocampal transplant; CA1/CA3-pyramidal neurons of the corresponding hippocampal fields; Y-young recipients; A-aged recipients. Bin-25 mkm. 
The distribution of neuronal sizes in grafts and in the hippocampi of aged recipients were drastically different. The unimodal distribution of the grafted neurons, with a maximum in the range of 325$400 \mathrm{mkm}$, differed from the bimodal distribution that was typical of the aged CA3 in situ and was similar to the histogram that was characteristic of young animals.

\section{DISCUSSION}

Changes of neuronal size in the in situ hippocampus during aging

Morphometric investigation of the pyramidal neurons in the fields CA1 and CA3 of the normal hippocampus in young and old animals was not a special aim of this investigation. On the one hand, however, such data were necessary for the detailed analysis of neuronal sizes in grafted tissue, and on the other hand, they were of special interest because a general agreement is lacking about potential changes of neuronal parameters during aging.

That $\mathrm{CA} 1$ and $\mathrm{CA} 3$ pyramidal neurons are selectively vulnerable to the action of various factors is well known. Thus, CAl neurons are sensitive to ischemia /29/, anoxia /7/ and to the effects of ethanol $/ 25 /$, whereas CA3 neurons suffer more under influence of hypothyroidism /27/ and corticosterone /19/. CAl pyramidal neurons have been shown to be selectively vulnerable in various pathological states in humans; the preferential death of CA1 neurons has been described in Alzheimer's disease $/ 33 /$, in Huntington's disease $/ 31 /$, in some forms of Parkinson's disease $/ 13 /$, as well as in schizophrenia $/ 2 /$.

The existing morphometric data on the influence of normal aging, however, are contradictory. The stability of neuronal morphometric parameters (neocortex) $/ 34 /$, as well as the decreased sizes of various neurons, including dentate granular cells $/ 5 /$ and cholinergic neurons of the anterior basal brain $16 /$, has been described for normal aging. Concerning the various signs of aging in the pyramidal neurons of hippocampal subfields, diametrical conclusions are found in the literature. Such signs have been variously reported to be expressed predominantly in CA $1 / 36 /, \mathrm{CA} 3 / 15 /$, or as absent in the hippocampus altogether, although present in the neighboring subicular complex and entorhinal cortex $/ 35 /$.

Our data reveal the differential effects of normal aging on CA1 and CA3 pyramidal cells. Whereas the morphometric somatic and nuclear parameters of the CA1 neurons were significantly decreased in the aged group of animals, no change of the mean sizes was detected in the CA3. We observed a complex redistribution of neuronal sizes, however, characterized by the appearance of two, approximately equal, neuronal populations with dimensions larger and smaller than those that are typical for CA3 neurons of young animals. Thus, our data confirm higher sensitivity of the CA1 pyramidal neurons to various detrimental factors, including normal aging of the brain. Concomitantly, the data also demonstrate the higher plastic adaptability of CA3 neurons, in which the atrophic changes of some neurons during the course of aging presumably are partly compensated by the hypertrophy of another subpopulation. The higher plasticity of the CA3 neurons thus correlates to the high expression of mRNA for GAP-43/16/ in this field of the adult hippocampus, whereas such mRNA is very low in CA1.

\section{Development of pyramidal neurons in hippocampal grafts}

Previous histological and electron microscopic investigations in our laboratory concerned the organization of hippocampal and septal grafts that were developing in the anterior eye chamber of adult rats $/ 28,37-39 /$. The finding that despite their isolation from other brain structures and the atypical innervation and vascularization provided by the host iris, grafts not only grow and develop but also reproduce the general morphology and cytoarchitectonics of the grafted brain region confirmed the histological data of other authors /12,24/.

The results of the present work extend this knowledge by demonstrating that the age of the host animal-at least during the first 45 days of graft development in oculo (the maximal period tested in this work)-does not exert an important influence on the survival and differentiation of pyramidal cells. This phenomenon has been shown in intrabrain grafts by other authors $/ 4,21,30,32 /$. The presence of a few vacuolized and dark neurons 
in certain grafts analyzed in the present study depended neither on graft rejection nor on the age of recipients, nor did it correlate to the size and general organization of the grafts. Some individual differences in cellular composition of the grafts also showed no correlation with the host age.

In both groups of recipients, morphometric analysis of the grafted neurons revealed a strong tendency toward increased mean sizes of CA1 neurons. No significant changes occurred in the parameters of CA3 neurons, except for the sizes of the pyramidal cell bodies that were grafted into young recipients. Even in this case, however, the effect was smaller (by half) than that in the CAl cells. In young animals, the distribution of the somatic sizes in situ and in oculo was the same.

Overall, we may state that in old recipients, the signs of senescence seen in the in situ hippocampus were not present in hippocampal grafts that were developing in their anterior eye chambers. The neuronal hypertrophy observed in the grafted tissue can be explained by several factors. A high level of metabolism, as demonstrated, for example, by the presence of coated vesicles, is characteristic of nervous tissue during ontogenetic development /1/ and regeneration /18/. An increased synthesis of neurotrophic factors has been described in both conditions $/ 20,22,23 /$. In our electron microscopic investigations of intraocular grafts, we also observed the ultrastructural signs not only of increased metabolic and transport processes /37-39/ but also of retarded maturation of nervous tissue 140/. Such a combination of immaturity and active regenerative processes might have caused the neuronal hypertrophy in the grafts.

Additionally, the absence of normal afferentation results in the hyperdevelopment of intrinsic connections between neurons, and this, in turn, may lead to an increased excitability of the cells, with easily provoked epileptiform discharges $/ 3,14 /$. Cellular hypertrophy may be caused by an increased level of activity. This interpretation of the results concerns only the CA1 neurons, however, because in CA3 a certain limited hypertrophy occurred only in young recipients, indicating an additional trophic influence of the young organism on the general growth of transplanted tissue $/ 8,28 /$.
Overall, the data presented here confirm that the CA1 and CA3 hippocampal pyramidal cells in intraocular grafts reproduce normal cytological and morphometric characteristics, expressing their genetic program in conditions of complete isolation from other brain structures. The CAl cells are more dependent on epigenetic, environmental factors and demonstrate greater changes during both normal aging and transplantation. The age of a recipient, however, had no significant influence on the development of the grafted cells in either hippocampal CA1 or CA3, and thus, cannot be regarded as a limiting factor for using nervous tissue transplantation to compensate for defective functions in the aging brain.

\section{ACKNOWLEDGMENTS}

The authors wish to thank A.G. Bragin and V.I. Hachko for methodical help. The work was supported by the Russian Foundation for Support of Basic Research (Project N 96-04-48537).

\section{REFERENCES}

1. Altman G. Coated vesicles and synaptogenesis. A developmental study in the cerebellar cortex of the rat. Brain Res 1971; 30: 311-322.

2. Benes FM, Sorensen I, Bird ED. Reduced neuronal size in the posterior hippocampus of schizophrenic patients. Schizophr Bull 1991; 17: 597-608

3. Bragin AG, Vinogradova OS. Comparison of neuronal activity in septal and hippocampal grafts developing in the anterior eye chamber of the rat. Dev Brain Res 1983; 10: 279-286.

4. Clarke DJ, Bjorklund A. Restoration of cholinergic circuitry in the hippocampus by foetal grafts. In: Frotscher M, Misgeld U, eds, Central Cholinergic Synaptic Transmission. Birkhauser Verlag, Basel. 1989; 275-287.

5. Coleman PD, Hornberger GC, Buell SI. Volume of the dentate gyrus in aging F344 rats. Neurosci Lett 1985; 22: 58 .

6. De Bilbao F, Jazat F, Lamour J, Senut MC. Agerelated chaa nges in galanin-immunoreactive cells of the rat medial septal area. J Comp Neurol 1991; 313: 613-624.

7. Dell'Anna ME, Calzolari S, Molinari M, Juvone L, Calimici R. Neonatal anoxia induces transitory hyper- 
activity, permanent spatial memory deficits and CAl cell density reduction in developing rats. Behav Brain Res 1991; 45: 125-134.

8. Eriksdotter-Nilsson M, Olson L. Growth of brain tissue grafts is dependent upon host age. Mech Ageing Dev 1989; 40: 1-22.

9. Fiandaca MS. Brain grafting for Parkinson's disease. Experimental, clinical, and immunological considerations. Transplantation 1991; 51: 549-556.

10. Gage FH. Fetal implants put to the test. Nature 1993; 361: 405-406.

11. Gash DM. Neural transplantation, potential therapy for Alzheimer's disease. J Neural Transmiss 1987; 524: 301-308.

12. Goldowitz D, Seiger A, Olson L. Anatomy of the isolated area dentata grown in the rat anterior eye chamber. J Comp Neurol 1982; 208: 382-400.

13. Hof PR, Perl DC, Loerzel AJ, Steele JC, Morrison JH. Amyotrophic lateral sclerosis and Parkinsonismdementia from Guam: Differences in neurofibrillary tangle distribution and density in the hippocampal formation and neocortex. Brain Res 1994; 656: $107-$ 116.

14. Hoffer BJ, Seiger A, Taylor D, Olson L, Freedman R. Seizures and related epileptiform activity in hippocampus transplanted to the anterior chamber of the eye. 1. Characterization of seizures, interictal spikes, and synchronous activity. Exp Neurol 1977; 54: 233250.

15. Kadar T, Silbermann M, Brandeis R, Levy A. Age-related structural changes in the rat hippocampus: correlation with working memory deficiency. Brain Res 1990; 512: 113-120.

16. Kruger L, Bendotti C, Rivolta K, Samanin R. GAP-43 mRNA localization in the rat hippocampus CA3 field. Mol Brain Res 1992; 13: 267-272.

17. Lindvall $O$. Prospects of transplantation in human neurodegenerative diseases. Trends Neurosci 1991; 14: 376-384.

18. Mc Williams RJ, Lynch G. Sprouting in the hippocampus is accompanied by an increase in coated vesicles. Brain Res 1981; 211: 158-164.

19. Magarinos AM, McEwen BS. Stress-induced atrophy of apical dendrites of hippocampal CA3 neurons: Involvement of glucocorticoid secretion and excitatory amino acid receptors. Neuroscience 1995; 69: 89-98.

20. Manthorpe $M$, Nieto-Sampedro $M$, Skaper SD, Lewis ER, Barbin G, Longo FM, Cotman CW, Varon S. Neuronotrophic activity in brain wounds of the developing rat. Correlation with implant survival in the wound cavity. Brain Res 1983; 267: 47-56.

21. Matsumoto A, Murakami S, Arai Y, Osanai M. Synaptogenesis in the neonatal preoptic area grafted into the aged brain. Brain Res 1985; 347: 363-367.

22. Needels DL, Nieto-Sampedro $M$, Cotman CW. Induction of a neurite-promoting factor in rat brain following injury or deafferentation. Neuroscience 1986; 18: 517-526.

23. Nieto-Sampedro $M$, Whittemore $S R$, Needels $D L$, Larson J, Cotman CW. The survival of brain transplants is enhanced by extracts from injured brain. Proc Natl Acad Sci USA. 1984; 81: 6250-6254.

24. Olson L, Freedman R, Seiger A, Hoffer B. Electrophysiology and cytology of hippocampal formation transplants in the anterior chamber of the eye. I. Intrinsic organization. Brain Res 1977; 119: 87-106.

25. Paula-Barbosa MM, Borges MM. Giant multivesicular bodies in the rat hippocampal pyramidal cells after chronic alcohol consumption. Neurosci Lett 1986; 64: 345-349

26. Pechanski M, Cesaro P. Hantraye P. Rationale for intrastriatal grafting of striatal neuroblasts in patients with Huntington's disease. Neuroscience 1995; 68: 273-285.

27. Rami A, Patel AJ, Rabie A. Thyroid hormone and development of the rat hippocampus: Morphological alterations in granule and pyramidal cells. Neuroscience 1987; 19: 1217-1226.

28. Saifullina VN, Vinogradova OS, Zhuravleva $Z N$, Bragin AG. Growth of the hippocampal grafts in the rat anterior eye chamber: Effects of age and strain of the recipients. J Hirnforschung 1990; 31: 505-513.

29. Schmidt R, Freund TF. Selective vulnerability of the hippocampus in brain ischemia. Neuroscience 1991; 40: 599-636.

30. Sladek JR, Gash DM. Morphological and functional properties of transplanted vasopressin neurons. In: Sladek JR, Gash DM, eds, Neural Transplants: Development and Function. New York-London, Plenum Press, 1984; 243-282.

31. Sprago E, Everall JP, Lantos PL. Neuronal loss in the hippocampus in Huntington's disease: A comparison with HIV infection. J Neurol Neurosur Psych 1993; 56: 487-491.

32. Steinbusch HWM, Beek A, Frankhuyzen AL, Tonnaer JADM, Gage FH, Bjorklund A. Functional activity of raphe neurons transplanted to the hippocampus and caudate-putamen. An immunohistochemical and analysis in adult and aged rats. Annals NY Acad Sci 1987; 495: 169-184.

33. Van Hoesen GW, Hyman BT. Hippocampal formation anatomy and the patterns of pathology in Alzheimer's disease. Brain Res 1990; 83: 445-457.

34. Vincent SL, Peters A, Tigges J. Effects of aging on the neurons within area 17 of Rhesus monkey cerebral cortex. Anat Rec 1989; 223: 329-341.

35. West MJ. Regionally specific loss of neurons in the aging human hippocampus. Neurobiol of Aging 1993; 14: 287-293.

36. Yang G, Wang E. Terminin (Tp63/60), a novel cell senescence-related protein, is present in the aging human hippocampus. Brain Res 1994; 644: 188-196. 
37. Zhuravleva ZN, Bragin AG, Vinogradova OS. Organization of the nervous tissue (hippocampus and septum) developing in the anterior eye chamber. I. General characteristic and non-neural elements. J Hirnforschung 1984; 25: 313-330.

38. Zhuravleva ZN, Bragin AG, Vinogradova OS. Organization of the nervous tissue (hippocampus and septum) developing in the anterior eye chamber. II. Neuronal perikarya and dendritic processes. J Hirnforschung 1985; 26:419-437.
39. Zhuravleva $\mathrm{ZN}$, Bragin AG, Vinogradova OS Organization of the nervous tissue (hippocampus and septum) developing in the anterior eye chamber. III. Axonal processes and their synaptic endings. J Hirnforschung 1986; 27: 323-341.

40. Zhuravleva ZN, Vinogradova OS, Bragin AG. Incomplete maturation of the nervous tissue transplanted into the anterior eye chamber. In: Trojan S, Stastny F, eds, Ontogenesis of the Brain. Universitas Carolina, Praha, 1986; 4: 67-70. 



Scientifica
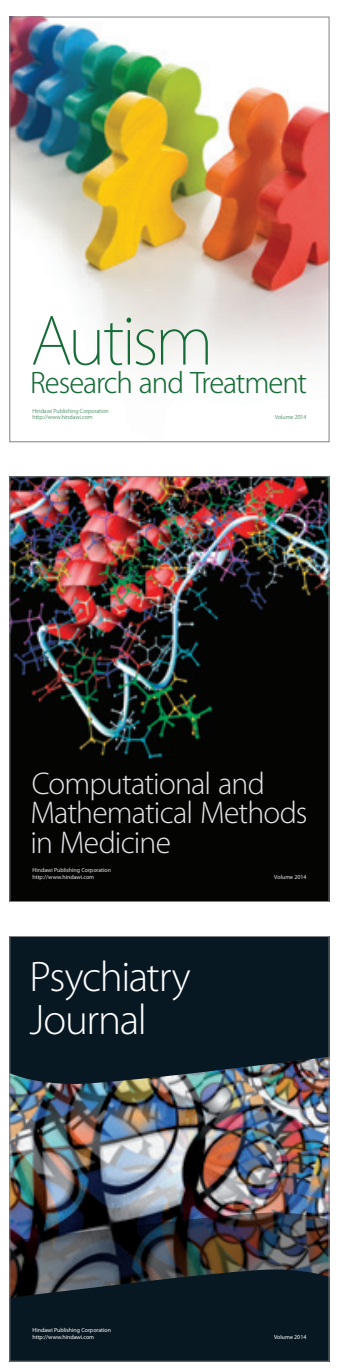
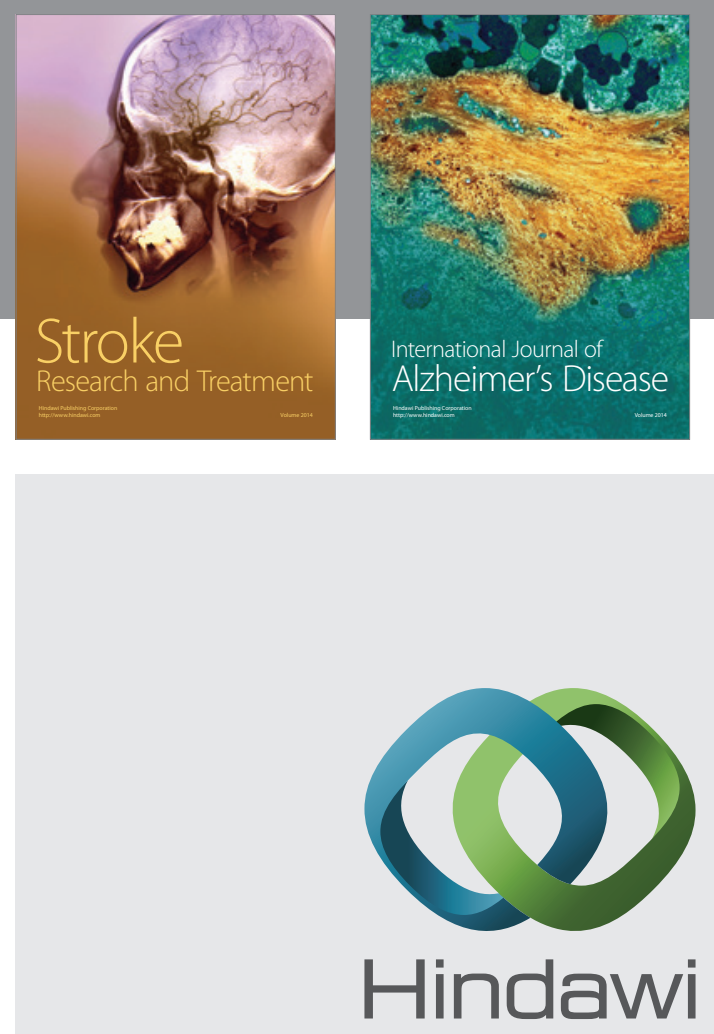

Submit your manuscripts at

http://www.hindawi.com
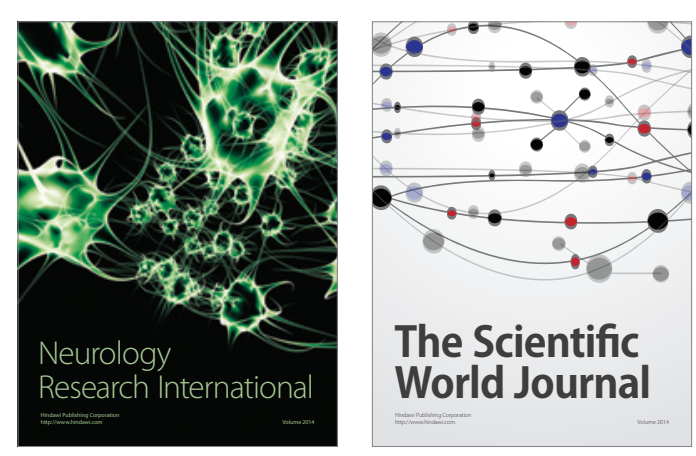

The Scientific World Journal

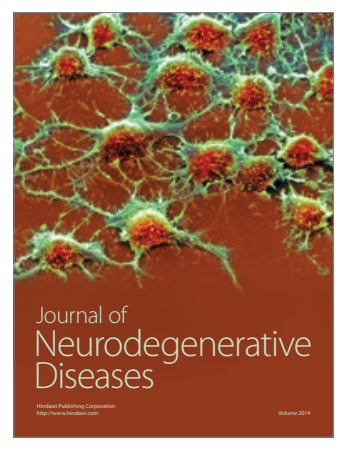

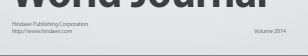

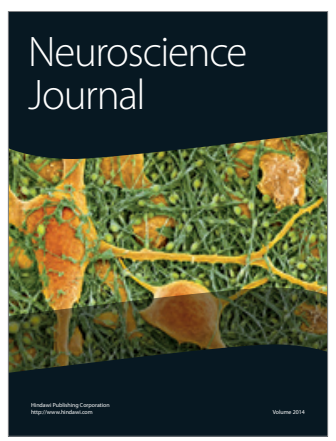

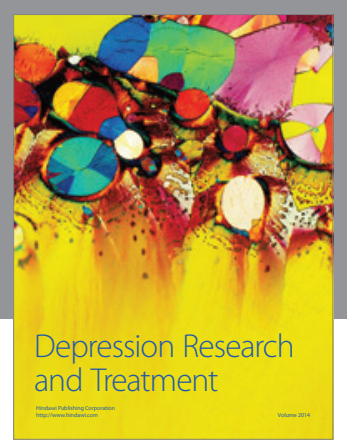
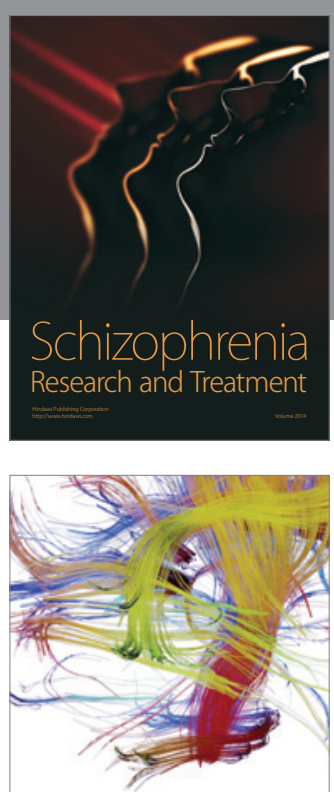

Brain Science

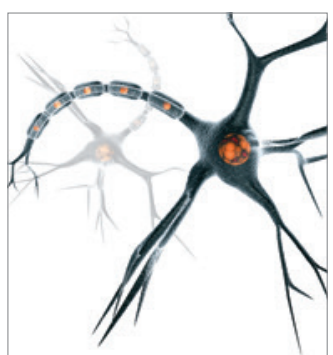

Neural Plasticity
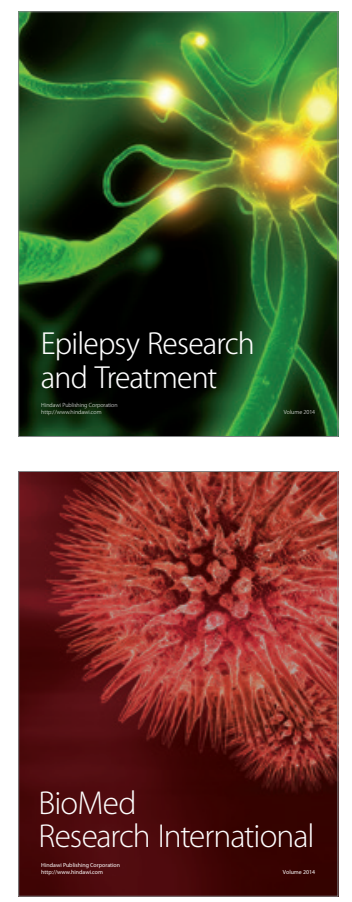

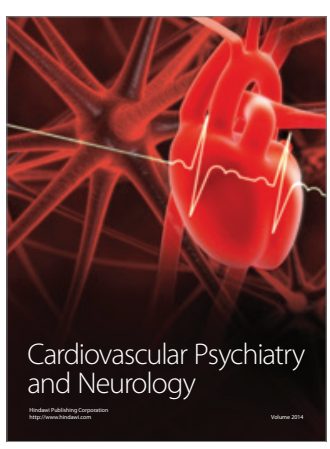

Parkinson's

Disease
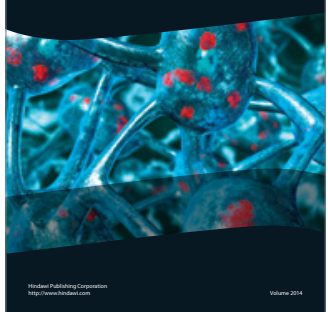